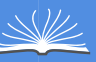

Global journals Inc.

(8)

(a)

Online ISSN: 2249-4618 \& Print ISSN: 0975-5888

\title{
Cosmetics and its Health Risks
}

By Jonathas Xavier Pereira \& Thaís Canuto Pereira Federal University of Rio de Janeiro (UFRJ)

Abstract- The use of cosmetic products is increasing around the world and a variety of chemical compounds used in the manufacture of these products grows at the same time. In this way, the risk of intoxication, allergic processes, prolonged chemical exposure, side effects and indiscriminate use are also increased. The present work aims to highlight the biological risks that cosmetics can pose to human health against the toxic substances used in its formulation. This work is an integrative literature review structured according to the methodology described by Whittemore, R. and Knafl, K., 2005. This work relates the main toxic chemical substances present in cosmetic products to the possible health complications reported in the scientific literature. Currently, the cosmetic industries have increased the use of compounds with preservative action, surfactant, fragrances, stains, etc. in the formulation of cosmetic products. Such substances potentiate the quality, property and shelf life of cosmetics, but on the other hand, many of these substances are toxic to the human body, presenting health risks ranging from a simple mild hypersensitivity reaction to an anaphylactic process or even a lethal intoxication. Thus, the indiscriminate use of cosmetics may present itself as an emerging issue of public health. In view of the above, this work seeks to encourage improvements in the search for new methodologies for quality control in the production and consumption of cosmetic products around the world.

Keywords: cosmetics, biological risks, toxicity, quality control, adverse effects.

GJMR-B Classification: NLMC Code: QT 275

Strictly as per the compliance and regulations of:

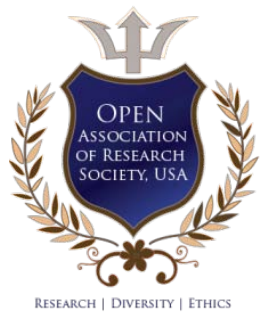

(C) 2018. Jonathas Xavier Pereira \& Thaís Canuto Pereira. This is a research/review paper, distributed under the terms of the Creative Commons Attribution-Noncommercial 3.0 Unported License http://creativecommons.org/licenses/by-nc/3.0/), permitting all non-commercial use, distribution, and reproduction in any medium, provided the original work is properly cited. 


\title{
Cosmetics and its Health Risks
}

\author{
Jonathas Xavier Pereira ${ }^{\alpha} \&$ Thaís Canuto Pereira ${ }^{\circ}$
}

Abstract- The use of cosmetic products is increasing around the world and a variety of chemical compounds used in the manufacture of these products grows at the same time. In this way, the risk of intoxication, allergic processes, prolonged chemical exposure, side effects and indiscriminate use are also increased. The present work aims to highlight the biological risks that cosmetics can pose to human health against the toxic substances used in its formulation. This work is an integrative literature review structured according to the methodology described by Whittemore, R. and Knafl, K., 2005. This work relates the main toxic chemical substances present in cosmetic products to the possible health complications reported in the scientific literature. Currently, the cosmetic industries have increased the use of compounds with preservative action, surfactant, fragrances, stains, etc. in the formulation of cosmetic products. Such substances potentiate the quality, property and shelf life of cosmetics, but on the other hand, many of these substances are toxic to the human body, presenting health risks ranging from a simple mild hypersensitivity reaction to an anaphylactic process or even a lethal intoxication. Thus, the indiscriminate use of cosmetics may present itself as an emerging issue of public health. In view of the above, this work seeks to encourage improvements in the search for new methodologies for quality control in the production and consumption of cosmetic products around the world.

Keywords: cosmetics, biological risks, toxicity, quality control, adverse effects.

\section{INTRODUCTION}

$\mathrm{H}$ istorically, cosmetics began to be used 6.000 years ago and its use has spread throughout the world. Such products had the purpose of adorning and perfuming the body, so as not to alter the structure and function of the skin. In ancient Egypt, records point to the use of cosmetics containing leadrich black pigments (Kohl) applied to the eye region. Lead in turn, when in contact with the skin, releases gaseous nitric oxide capable of activating the immune system through vasodilation and activation of macrophages with cytotoxic characteristics [1].

Cosmetics and skin care products are currently consumed worldwide, with frequent use, increasing the exposure of the human body to the various chemical compounds that make up its formulas. Stipulating the incidence of cosmetic side effects is quite difficult because users with weak side effects often do not seek medical advice $[1,2]$.

Author a: Pereira, J.X. Department of Pathology; Faculty of Medicine; Federal University of Rio de Janeiro (UFRJ); Rio de Janeiro, Brazil. e-mail: jonathasxp@gmail.com.

Author o: Pereira, T.C. Biomedical Sciences Institute; Federal University of Rio de Janeiro (UFRJ); Rio de Janeiro, Brazil.

e-mail: canutothais@gmail.com
The side effects derived from the use of cosmetics pose health risks mainly due to exposure to numerous chemical substances. Its consequences can range from a simple mild hypersensitivity reaction to an anaphylactic process or even a lethal intoxication [1-3].

a) The beginning of complications due to the use of cosmetics

The use of pigmented lead-based cosmetics used by Egyptians is characterized as the earliest evidence of the use of cosmetics and their complications. Subsequently, rouges and lipsticks appeared whose reddish coloration was attributed to mercury sulfide. Such a compound, when ingested by pregnant women, caused miscarriage spontaneously. Another toxic compound capable of causing damage to the organism was the arsenic used by Greeks and Romans in chemical depilatory solutions [1].

With the advancement of knowledge about the physiology of skin and its components, the pharmaceutical industries started to invest in new active principles and vehicles for the production of cosmetics. Thus, new quality control tests in the manufacture of such cosmetics must be also updated in order to ensure safety in the use of such compounds [4].

Several regulatory agencies around the world are dedicated to the control and regulation of commercial activities, safety and quality control of cosmetics. Although there are rules and quality control tests to be followed for the manufacture of a cosmetic, these regulatory mechanisms are not fully effective, as the adverse effects still persist in the cosmetic consumer population. [2].

\section{b) The cosmetic risk and the public health}

In front of the arising use of cosmetic products and the larger exposure to the formulas compounds for a large time and frequency, the side effects of these products become more frequent in the population around the world. Women and men all over the world use large amount of cosmetic products in pursuit of everlasting youth, ignoring the probable health risks [5].

Cosmetic ingredients are emerging pollutants too. Their environmental monitoring is at a very early stage. However, it is known that they reach the environment in multiple ways, often through water, posing health risks to marine and freshwater ecosystems and to humans [5].

Thus, in public health science, the term "cosmetovigilance" began to represent a kind of health surveillance where the aim is the safety of the cosmetic 
product for commercial purposes. This surveillance is very important to control potentially hazardous ingredients and can thus set our minds at ease on the products placed on the market [6].

The restrictions on the use of some cosmetic ingredients are stipulated by several health surveillance agencies around the world mean that any ingredient not on the restriction's list be allowed. Thus, as the industry is quite creative and is always looking to improve its products, it is constantly using new ingredients not listed in the restriction's list. Such ingredients are new potential allergens. Unlike medicines, there is no a specific agency to assess the safety of cosmetic products, no marketing authorization with specific requirements, no evaluation of the risk-benefit ratio and no guarantee of constancy from one batch to another [6].

The health risks associated with the use of cosmetic products become currently an emerging public health problem, where about $12 \%$ of users in the general population had experienced undesirable effects with one or several cosmetic products in the last nine years $[5,6]$.

\section{il. Methodology}

This study is an integrative literature review based on the model proposed by Whittemore and Knafl, where it aims to relate clinical and toxicological aspects in the use of cosmetic products [7]. This review has selected a total of 32 studies published between 1998 and 2015, derived from the PubMed database, as shown in Figure 1.

\section{Distribution of selected articles}

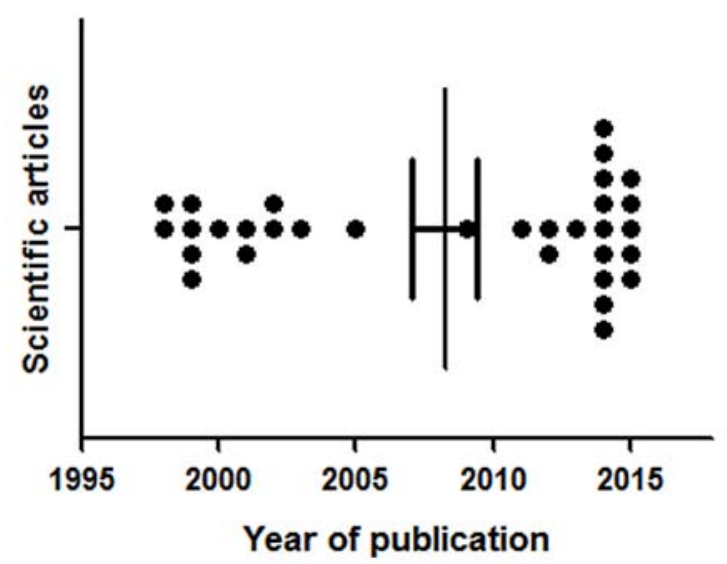

Fig. 1: Distribution of selected articles by year of publication. The graph shows the average year of publication and the standard deviation of the mean

The scientific articles followed the inclusion criteria: articles that contain the descriptors "cosmetics toxicity"; "cosmetic intoxication"; "cosmetic risk"; "cosmetic danger"; "cosmetic side effects".

The scientific articles followed the exclusion criteria: articles related to cosmetic surgery or that do not relate to cosmetic products were excluded.

The selection of scientific articles included reviews and original articles with a toxicological and clinical approach. This methodology aims to relate the main toxic chemical substances present in cosmetic products to the possible health complications reported in the scientific literature. Through this integrative study, the clinical-toxicological correlation becomes a valuable instrument to clarify and understand the side effects in using cosmetics, drawing attention to the neglected use of these products and highlighting the associated health risks.

\section{ili. Substances with Toxic Potential in the Formulation of Cosmetics}

In face of technological innovations in the cosmetics industry, many of these products have been added with chemical additives to increase their performance, effectiveness and viability [8]. Some examples of these chemical additives are Diazolidinyl Urea, Dioxane, Formaldehyde and Paraformaldehyde, Imidazolidinyl urea, heavy metals, Methylchloroisothiazolinone-methylisothiazolinone (MCl-Ml), Methyldibromoglutaronitrile-phenoxyethanol (MDBGN-PE), Parabens, Phthalate, Quaternium-15, Thimerosal and others.

\section{a) Diazolidinyl Urea}

It is an additive used since 1982 in the manufacture of personal care products such as child care products, eye and face make-up, skin care products, hair and nails [9]. Figure 2 shows its chemical formula.

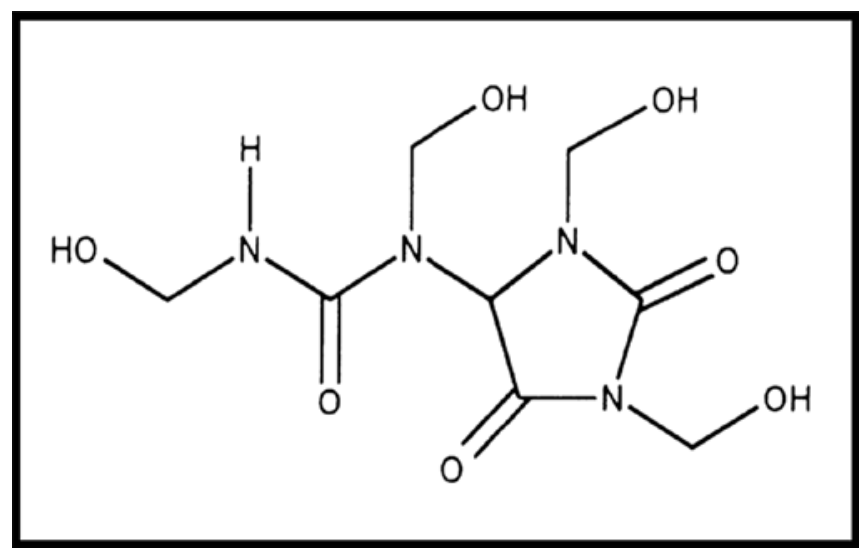

Fig. 2: Chemical formula of Diazolidinyl Urea

Exposure to this compound is capable of causing allergic contact dermatitis, as well as being characterized as a mutagenic and carcinogenic agent since it is capable of releasing formaldehyde, a fixative and preservative which will be discussed later [9]. 
b) Dioxane

1,4-dioxane (Figure 3) is an ether with an emulsifying, detergent and solvent function commonly found in products such as shampoo, toothpaste and mouthwash [8].

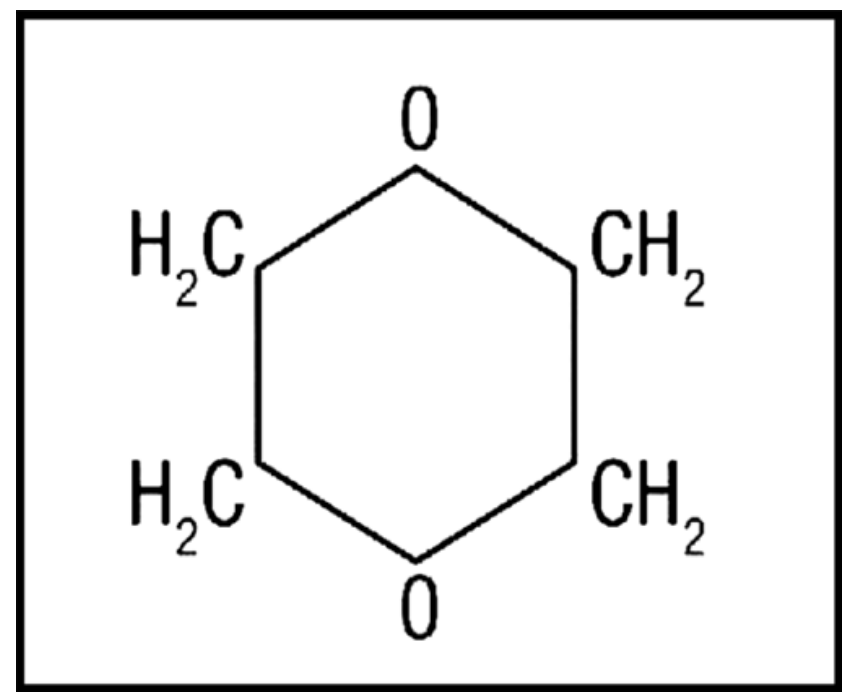

Fig. 3: Chemical formula of 1,4-Dioxane

Although this compound is not listed as a cosmetic component, this substance is a contaminant in the manufacturing ethoxylation step, creating other ingredients such as polyethylene glycol, polyethylene and polyoxyethylene. Thus, high levels of this contaminant can be observed in cosmetic products, being such chemical substance a potent carcinogen, capable of triggering cancer of breast, skin and liver. [8].

\section{c) Formaldehyde and Paraformaldehyde}

Formaldehyde and paraformaldehyde are toxic preservatives, the paraformaldehyde being a polymer derived from formaldehyde (Figure 4) [10].<smiles>C=O</smiles>

Formaldehyde<smiles>COC(C)C</smiles>

Paraformaldehyde
Fig. 4: Chemical formula of Formaldehyde and Paraformaldehyde

Formaldehyde has molecular characteristics that result in an agent of great potential risk to cancer [9]. Clinical studies show that 13\% of a sample of 957 participants had allergic contact dermatitis caused by formaldehyde, which is the second largest cause of contact dermatitis from cosmetic products [11].

In a study conducted by Agner, et al., 1999, 57 patients were evaluated for their exposure to formaldehyde. For this analysis, the participants were asked to bring the main cosmetics that were used by them on a daily basis. In total, 409 products were cataloged, and among them, 103 had formaldehyde in their compositions [12].

\section{d) Imidazolidinyl Urea}

Imidazolidinyl urea (Figure 5) is a preservative used in cosmetic products which also has the property of releasing formaldehyde as a consequence of its degradation, like Diazolidinyl Urea [3,13].

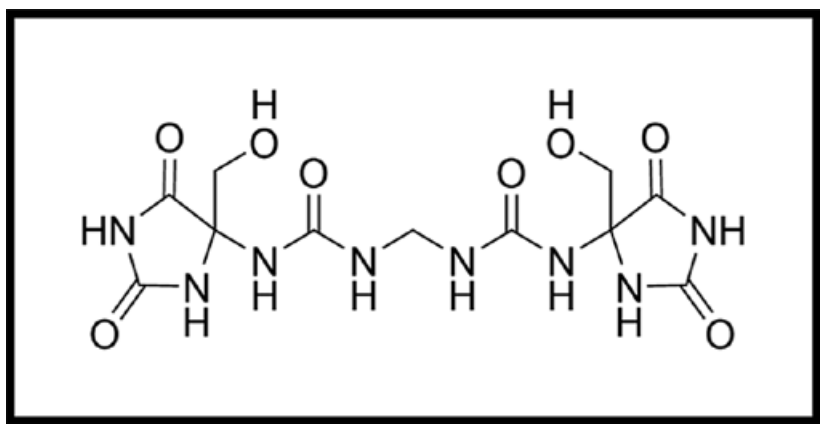

Fig. 5: Chemical formula of Imidazolidinyl Urea

Concentrations of $0.01 \%$ to $1 \%$ of Imidazolidinyl urea at 24 hours in contact with a culture of cells from human peripheral blood were considered moderately cytotoxic doses. At concentrations of $0.1 \%$ to $0.5 \%$, the same effect was observed in only 3 hours [14].

Clinical trials point to Imidazolidinyl urea as a causative agent of contact dermatitis allergies $[3,11,15]$.

\section{e) Heavy metals}

A group of hazardous substances in the manufacture of cosmetics is toxic heavy metals, such as lead $(\mathrm{Pb})$, cadmium $(\mathrm{Cd})$, nickel $(\mathrm{Ni})$, arsenic $(\mathrm{As})$ and mercury $(\mathrm{Hg})$. Some cosmetics may contain aluminum (Al), classified as a light metal. As there is no single and effective regulation around the world, some cosmetics like colorful cosmetics, face and body care products, hair cosmetics and herbal cosmetics may contain in their formulation relatively high amounts of these heavy metals. These elements can accumulate on the skin and internal organs, causing toxic effects that can be classified into topical (mainly contact dermatitis), and systemic (systemic allergic dermatitis) [16].

Some metals serve as pigment substances, for example, chromium ( $\mathrm{Cr}$ ) used mainly eyeshadows and blushes. Pigmented cosmetics of a reddish color, for example, are commonly contaminated with arsenic (As), Lead $(\mathrm{Pb})$ e mercury $(\mathrm{Hg})$ [17].

Antimony (Sb) can cause pneumoconiosis, alterations in pulmonary function, bronchitis, emphysema, abdominal pain, vomiting, diarrhea and ulcers. This metal is mainly found in lipsticks, eye pencils and face powder. Arsenic (As) can cause skin disorders, circulatory and peripheral nervous disorders, an increased risk of lung cancer, and a possible 
increase in the risk of the gastrointestinal tract and the urinary system cancers. This metal is mainly found in make-up powder and skin cream. Cadmium (Cd) may accumulate in the kidneys, with possible damage. Chronic exposure to low levels of cadmium can also cause bone fragility and consequent bone fractures. Cadmium is commonly found in hair creams, lipsticks and skin cream. The Chromium $(\mathrm{Cr})$ in its oxidized state can cause contact allergies. Its presence in cosmetics is mainly associated with eyeliner, eye pencil, eyeshadow, lipstick and make-up powder. Cobalt (Co) and nickel (Ni) can cause allergies such as contact dermatitis, and these metals are commonly present in cosmetics such as eye shadow, face paint, hair cream and lipstick. The lead $(\mathrm{Pb})$, when ingested in large quantities, may interfere with the synthesis of hemoglobin and calcium channels, whose functions are important for nerve conduction. Lead is found in dyes for hair (such as lead acetate whose chemical formula is shown in Figure 6) and lipsticks, eyeliner, eye pencil, hair cream in their inorganic form, and can be minimally absorbed by the skin $[8,17]$.

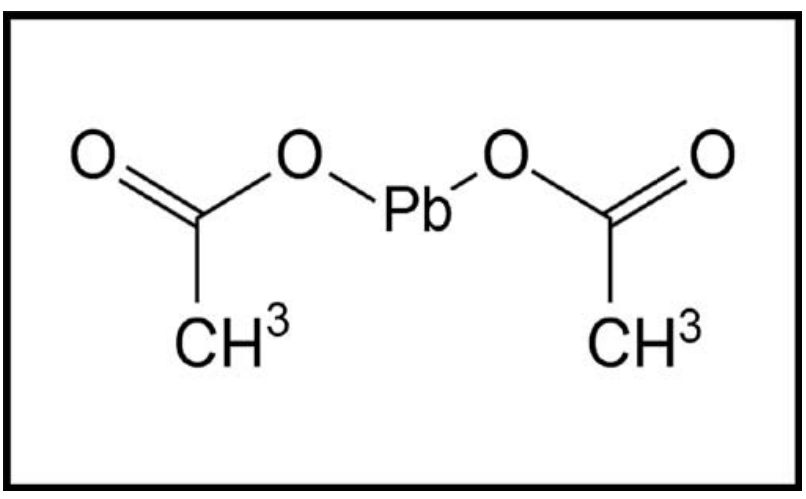

Fig. 6: Chemical formula of Lead acetate

f) Methylchloroisothiazolinone-methylisothiazolinone (MCl-MI)

$\mathrm{MCl}-\mathrm{Ml}$ (Figure 7) is a preservative widely used in the manufacture of personal hygiene products, which has a high degree of cytotoxicity and is dose-dependent when tested in vitro, in cultured cells $[14,18]$. In cosmetic products, this compound represents an important allergen and may cause hypersensitivity when there is constant exposure to this substance. [19].<smiles>Cn1sc(Cl)cc1=O</smiles>

Methylchloroisothiazolinone

Fig. 7: Chemical formulas of Methylchloroisothiazolinone-methylisothiazolinone (MCl-MI)

\section{g) Methyldibromoglutaronitrile-phenoxyethanol} (MDBGN-PE)

MDBGN-PE (Figure 8) is also a preservative used in the manufacture of cosmetics such as, for example, moisturizing creams. This compound is the preservative with the highest potential to cause contact dermatitis, presenting an average of $14.5 \%$ incidence on the population $[19,20]$.

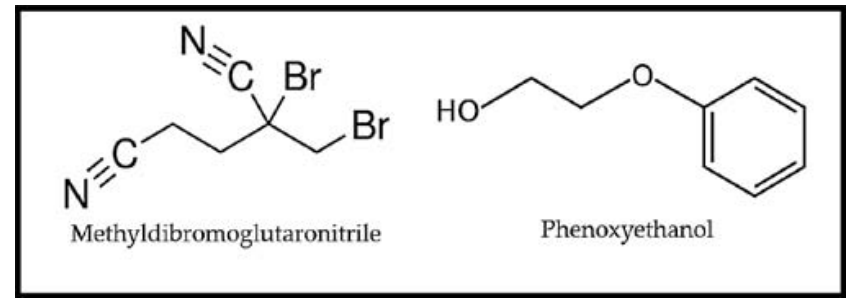

Fig. 8: Chemical formulas of Methyldibromoglutaronitrile-phenoxyethanol (MDBGN-PE)

\section{h) Parabens}

Parabens are esters of p-hydroxybenzoic acid, with alkyl substituents ranging from methyl to butyl or benzyl groups. Parabens are also a group of substances for the purpose of preservation. Some examples of these chemical additives are methylparaben, ethylparaben, propylparaben, butylparaben and benzylparaben (Figure 9). Its use is also associated with antimicrobial action and is widely used in the cosmetics industry because it has a low cost for its implementation in the production. Methylparaben and propylparaben are the most commonly used and often present in the cosmetic products together $[21,22]$.

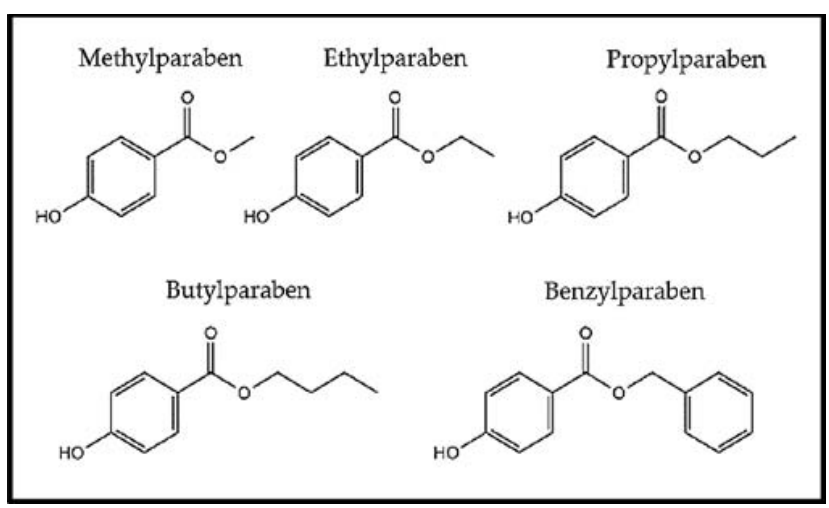

Fig. 9: Chemical formulas of Parabens

In the past, the use of parabens in higher concentration triggered allergic reactions in the population. Nowadays, in conjunction with the change of safety resolutions for cosmetics and hygiene and beauty products, the use of parabens has become very low, being used in low concentrations, which reflects in a reduction in the positive cases related to allergic reactions or contact dermatitis [23].

\section{i) Phthalate}

Phthalate esters are derivatives of phthalic acid and often found in products as plasticizers, solvents, 
and alcohol denaturants. Phthalate compounds may be found in a diverse number of cosmetics (nail polish, lotions, hair care products, perfumes). The most commonly encountered forms are dimethyl phthalate and diethyl phthalate, and their chemical formulas are shown in Figure 10 [8].<smiles>COC(=O)c1ccccc1C(=O)OC</smiles>

Dimethyl Phthalate<smiles>CCOC(=O)c1ccccc1C(=O)OCC</smiles>

Diethyl Phthalate

Fig. 10: Chemical formulas of Dimethyl phthalate and Diethyl phthalate

Exposure to these components can trigger developmental disorders and breast cancer. Some experimental studies have shown that high levels of phthalate could change hormone levels and cause genital-related birth defects in rodents [8].

\section{j) Quaternium-15}

Quaternium-15 (Figure 11) is a common contact allergen included in the European baseline series. The prevalence of contact sensitization is as low as 1.2$1.6 \%$, but could represent the first event leading to systemic anaphylactic reaction through a switch from type IV to type I allergy [24].

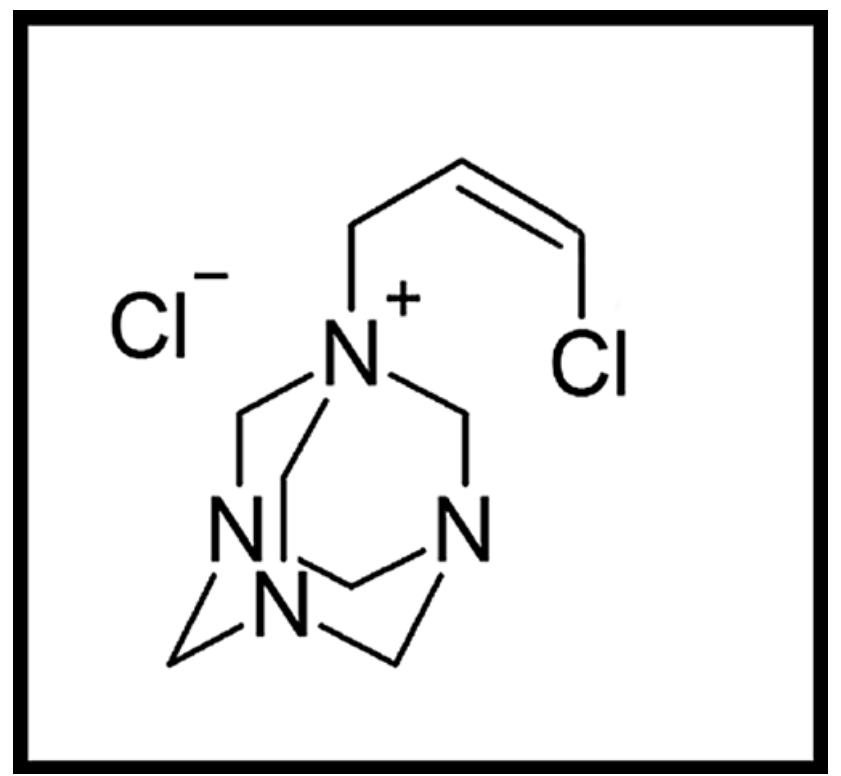

Fig. 11: Chemical formula of Quaternium-15

\section{k) Thimerosal}

Thimerosal (Figure 12), a mercuric derivative of the thiosalicylic acid, is a preservative used in several types of consumer products, including cosmetics, ophthalmic and otolaryngologic medications, and vaccines [26].

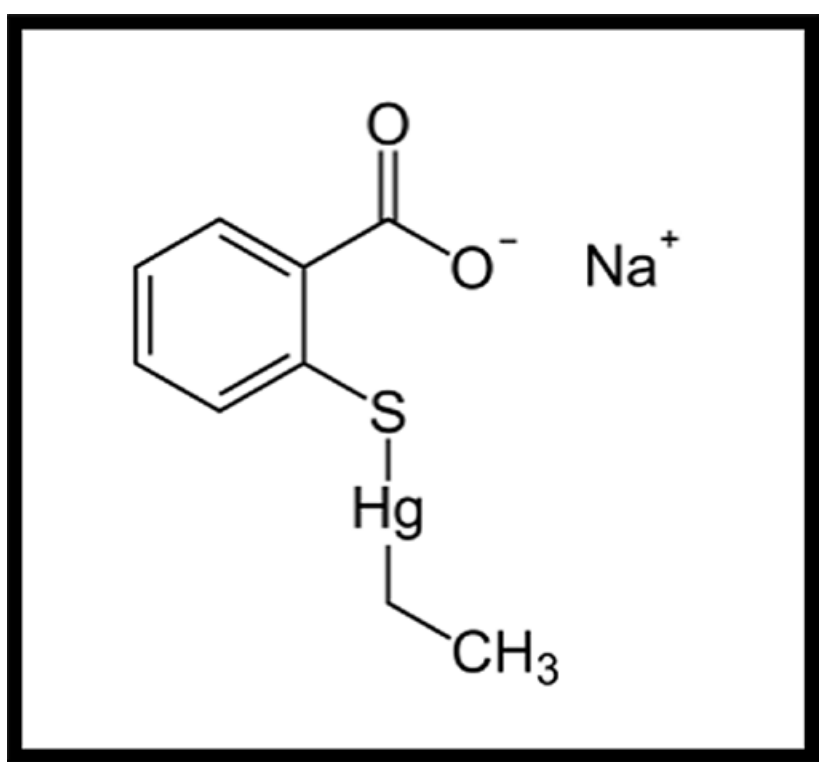

Fig. 12: Chemical formula of Thimerosal

Due to a large number of allergic reactions and environmental problems, its use has been diminished in the last two decades [26]. Thimerosal is used primarily for the conservation of eye shadows, make-up removers, masks, and soap-free cleansers [27].

\section{I) Others substances}

Although the preservatives mentioned above are the substances responsible for most adverse effects from cosmetics, other substances have allergenic potential, for example:

- Fragrances (Myroxylon Pereirae);

- Chemical contamination to obtain surfactants (Cocamidopropyl betaine) used in the production of shampoo, liquid soap, skin cleansers, shower gels, and deodorants;

- Oxidizing agents (Paraphenylenediamine) very common in hair dye composition;

- Glyceryl monothioglycolate, used in permanent hair solutions (to be curled or waved hair);

- Toluenesulfonamide-formaldehyde resin, used in the manufacture of nail polishes and lacquers;

- Propyl gallate, octyl gallate, and dodecyl gallate are all antioxidants used to prevent the deterioration of unsaturated fatty acids that can cause discoloration and odor, present in the composition of cosmetic creams and lotions [25,28].

\section{Possible Health Complications Associated WITH THE USE OF Cosmetics}

Due to the presence of numerous components in the formulation of cosmetic products, such products 
have the potential to cause side effects and their consequences can range from a simple mild hypersensitivity reaction to an anaphylactic process or even a lethal intoxication [1-3].

There are many types of adverse reactions caused by cosmetics. Most adverse reactions are irritant, however, type IV hypersensitivity, contact urticaria, photosensitization, pigmentary disorders, damage of hair and nails, paronychia, acneiform eruptions, folliculitis, and exacerbation of an established dermatosis may also occur. Side effects of cosmetic products do occur. It is to be expected that the improvements in safety, tolerance, and skin compatibility will not prevent side effects to cosmetic products from increasing in the future because of the continuing goals to intensify their biological activity and therapeutic efficacy. [25,29].

The areas of the body most affected by adverse reactions attributed to the use of cosmetics are head and neck, and irritant dermatitis is the most common type of complication $[25,29]$. The health complications associated with the use of cosmetic products can be:

\section{a) Allergic reactions to cosmetics}

Allergic reactions to cosmetics constitute a small but significant portion of the complications associated with the use of cosmetics. Allergic contact dermatitis represents true delayed-type (type IV) hypersensitivity that presents eczematous dermatitis and comprises approximately $10 \%$ to $20 \%$ of all cases of contact dermatitis. Type IV is hypersensitivity reaction that is T-cell mediated, wherein circulating or resident sensitized $T$ cells are activated by the offending allergen to release pro-inflammatory cytokines. Sensitization depends on several factors including product composition, a concentration of potential allergenic components, amount of product applied, site application, skin barrier integrity, and frequency and duration of application $[2,19,25,29]$.

This clinical scenario can range from mild erythema and scale with a minimal itch to vesicular, bullous, indurated plaques that are intensely pruritic. Initial sensitization is required for the subsequent expansion of a reaction when exposure occurs again [19].

Allergic contact urticaria is immediate-type hypersensitivity that represents a true allergic reaction. As the name implies, the reactions occur within minutes to hours and might be limited to the site of exposure on the skin or, in severe cases, reactions can be generalized. Contact urticaria is a rarer reaction to cosmetics and skin care products that may be an immunologic or nonimmunologic reaction. It is characterized by the development of a wheal-and-flare response to a topically applied chemical. The spectrum of clinical presentation ranges from itching and burning to generalized urticaria to anaphylaxis. In highly allergic individuals, mucosal exposures, or large exposures, the symptoms of immediate-type hypersensitivity can generalize and include conjunctivitis, cough, bronchoconstriction, hypotension, anaphylaxis, and, occasionally, death. $[2,3,19]$.

\section{b) Irritant contact dermatitis}

This is the most commonly encountered type of complication due to the use of cosmetics, especially those containing methylchloroisothiazolinonemethylisothiazolinone (MCl-MI) in its formulation. There are currently over 57.000 described irritants worldwide, ranging from weak or marginal irritants to strongly corrosive acids and bases. Then, the majority of facial problems that arise with skin care products and cosmetics are of the irritant contact dermatitis type manifesting as erythematous, burning, pruritic skin that may develop microvesiculation and later desquamation. The dermatitis is characterized by stratum corneum damage without immunologic reaction $[3,19]$.

Facial irritant dermatitis, which results mostly from cosmetics, presents as papules and plaques. Another common presentation is a "seborrheic-like dermatitis" with pink scaly plaques on the cheeks and chin. Less commonly, patients may develop urticarial or infiltrated plaques [28]

\section{c) Photoallergic dermatitis}

This type of allergic reaction occurs after contact with cosmetic products and subsequent exposure to light. Usually, such a reaction presents itself as sunburn that may be followed by hyperpigmentation and desquamation. This reaction is formed by chemical substances capable of absorbing radiation, especially ultraviolet $A$, in addition to having no definite immune mechanism. Its clinical manifestations vary from erythema, edema to vesiculation. The incidence of photoallergic dermatitis is low, and is mainly caused by fragrances methylcoumarin and musk ambrette, antibacterial agents and the para-aminobenzoic acid esters as sunscreening agents [3].

Photoallergy is an uncommon acquired altered reactivity dependent on an immediate antibody or a delayed cell-mediated reaction [30].

\section{d) Facial stinging}

There is a group of patients who note stinging or burning within several minutes after applying a cosmetic that intensifies over 5 to 10 minutes and then resolves after 15 minutes. This effect occurs without the patient exhibiting allergic contact dermatitis or irritant contact dermatitis with the applied substance. Tests should be done on the skin of the patient before using such components. Usually, substances such as benzene, phenol, salicylic acid, resorcinol and phosphoric acid are the main cause of facial stinging [3]. 


\section{e) Redness}

The redness of the skin caused by cosmetic products, especially soaps, is associated with the unbalance in cutaneous $\mathrm{pH}$. Modern soap is a blend of tallow and nut oil, or the fatty acids derived from these products, in a ratio of $4: 1$. This fact allows the $\mathrm{pH}$ of these soaps to be commonly alkalized ( $\mathrm{pH}$ 9-10), which can generate redness in the skin, which normally has a $\mathrm{pH}$ of 5.2-5.4. Ideally, such compounds should have neutral or slightly acidified $\mathrm{pH}$ [3].

Another reason redness can occur is the use of moisturizers with a greater oily proportion, allowing skin warming throughout the day [3].

\section{Results}

Today, innovation, research and development of new cosmetic products have increased the use of compounds with preservative action, surfactant, fragrances, stains, etc. Such substances enhance the quality, property and shelf life of cosmetic formulations, but on the other hand, many of these substances can be harmful to human health because of frequent, prolonged and indiscriminate exposure.

There are several agencies around the world to regulate the quality control, safety and production of cosmetic products, which are responsible for adjusting the standards and guidelines for the safe and healthy use of such products by the population, minimizing health risks. However, there is no specific agency that regulates the cost-benefit, guarantee of safety in the use of substances with toxic potential when applied to cosmetic products.

This work reviewed several substances with potential toxic to the human body, which can be found in the formulation of various cosmetic products around the world. We also reviewed the possible health complications reported by the scientific literature that are associated with the use of cosmetics and attributed to such toxic substances.

The scientific literature reveals that high amounts of chemical preservatives, perfumes and emulsifiers used in the manufacture of cosmetic products increase side efects and health risks through chemical and physical principles [31].

Chemically, preservative compounds generally have chemical structures associated with aromatic rings which generally have toxic potential, and ability to bind to metal elements that promote bioaccumulation in the body [32]. Although not all compounds that present toxic potential have their toxicity mechanism clarified in the literature, clinical evidence obtained by the side effects after the use of these substances point to the health risk associated with the use of cosmetics. The health risk associated with the use of cosmetics can ranging from a simple mild hypersensitivity reaction to an anaphylactic process or even a lethal intoxication.
Cancer is also a complication associated with the use of cosmetics in the face of clinical evidence reported in the literature [33].

Faced with the occurrence of side effects, the imminence of complications associated with the use of cosmetics contribute to an emerging public health problem, it is concluded that the process of quality control in the manufacture of cosmetic products is not completely effective in preventing health risks associated with the use of cosmetic products.

\section{Vi. Conclusion}

The cosmetic products may present health risks and recurrent adverse effects are attributed to the toxic substances commonly found in their formulations. Although the various structures for the regulation and quality control of cosmetics around the world are quite complex and comprehensive, they should be more rigorous in the inclusion of new substances with toxic potential in the formulation of cosmetics to avoid damages to human health.

To encourage improvements in the manufacture, marketing and use of cosmetic products by the population, it is necessary to apply a unified cosmetovigilance around the world. This public health strategy are a genuine means of obtaining information on the safety of cosmetic products and their ingredients, preventing the risks associated with using cosmetics become a serious public health problem.

\section{AcKnOWledgments}

The authors thanks Prof. Márcia Cury El-Cheikh for encouraging independent publications and for stimulating the professional career of her students. The authors thanks Dr. Yuri Cavalcante for the critical review and suggestions for the completion of this review article. The authors thanks Prof. Mariana Pereira Cabanel and Prof. Sergio Lisboa Machado for the support and intellectual incentive. The authors thanks the Federal University of Rio de Janeiro (UFRJ) for the professional reception.

\section{Conflict of Interest}

The authors state that there is no conflict of interest.

\section{References Références Referencias}

1. Draelos ZD. Cosmetics: The Medicine of Beauty. J Cosmet Dermatol.14 (2):91 (2015)

2. Alani Jl, Davis MDP, Yiannias JA. Allergy to cosmetics: A literature review. Dermatitis. 24(6):283-90 (2013).

3. Draelos ZD. Facial skin care products and cosmetics. Clin Dermatol [Internet]. 32(6):809-12 (2014).

4. Dreno B, Araviiskaia E, Berardesca E, Bieber T, Hawk J, Sanchez-Viera M, et al. The science of 
dermocosmetics and its role in dermatology. J Eur Acad Dermatology Venereol. 28(11):1409-17 (2014)

5. Nicolopoulou-Stamati P, Hens L, Sasco AJ. Cosmetics as endocrine disruptors: are they a health risk? Rev Endocr Metab Disord. 16(4):373-83 (2015).

6. Vigan M, Castelain F. Cosmetovigilance: definition, regulation and use "in practice." Eur J Dermatology. 24(6):643-9 (2014).

7. Whittemore $R$, Knafl $K$. The integrative review: Updated methodology. J Adv Nurs. 52(5):546-53 (2005).

8. Juhász MLW, Marmur ES. A review of selected chemical additives in cosmetic products. Dermatol Ther. 27(6):317-22 (2014).

9. Pfuhler S, Wolf HU. Effects of the formaldehyde releasing preservatives dimethylol urea and diazolidinyl urea in several short-term genotoxicity tests. Mutat Res - Genet Toxicol Environ Mutagen. 514(1-2):133-46 (2002).

10. Lv C, Hou J, Xie W, Cheng $H$. Investigation on formaldehyde release from preservatives in cosmetics. Int J Cosmet Sci. 37(5):474-8 (2015).

11. Warshaw EM, Buchholz HJ, Belsito D V., Maibach HI, Fowler JF, Rietschel RL, et al. Allergic patch test reactions associated with cosmetics: Retrospective analysis of cross-sectional data from the North American Contact Dermatitis Group, 2001-2004. J Am Acad Dermatol [Internet]. 60(1):23-38 (2009).

12. Agner T, Flyvholm MA, Menne T. Formaldehyde allergy: a follow-up study. Am J Contact Dermat. 10(1):12-7 (1999).

13. Doi T, Takeda A, Asada A, Kajimura K. Characterization of the decomposition of compounds derived from imidazolidinyl urea in cosmetics and patch test materials. Contact Dermatitis. 67(5):284-92 (2012).

14. Anselmi C, Ettorre A, Andreassi M, Centini M, Neri $P$, Di Stefano A. In vitro induction of apoptosis vs. necrosis by widely used preservatives: 2Phenoxyethanol, a mixture of isothiazolinones, imidazolidinyl urea and 1,2-pentanediol. Biochem Pharmacol. 63(3):437-53 (2002).

15. Katz AS, Sherertz EF. Facial dermatitis: Patch test results and final diagnoses. Am J Contact Dermat. 10(3):153-6 (1999).

16. Borowska S, Brzóska MM. Metals in cosmetics: Implications for human health. J Appl Toxicol. 35(6):551-72 (2015).

17. Bocca B, Pino A, Alimonti A, Forte G. Toxic metals contained in cosmetics: A status report. Regul Toxicol Pharmacol [Internet]. 68(3):447-67 (2014).

18. Smith CN, Alexander BR. The relative cytotoxicity of personal care preservative systems in Balb/C 3T3 clone A31 embryonic mouse cells and the effect of selected preservative systems upon the toxicity of a standard rinse-off formulation. Toxicol Vitr. 19(7):963-9 (2005).

19. Cohen DE, Kaufmann JM. Hypersensitivity reactions to products and devices in plastic surgery. Facial Plast Surg Clin North Am. 11(2):253-65 (2003).

20. Jackson JM, Fowler JF. Methyldibromoglutaronitrile (Euxyl K400): A new and important sensitizer in the United States? J Am Acad Dermatol. 38(6 I):934-7 (1998).

21. Mowad CM. Allergic contact dermatitis caused by parabens: 2 case reports and a review. Am J Contact Dermat [Internet]. 11(1):53-6 (2000).

22. Błedzka D, Gromadzińska J, Wasowicz W. Parabens. From environmental studies to human health. Environ Int. 67:27-42 (2014).

23. Sertoli A, Francalanci S, Acciai MC, Gola M. Epidemiological survey of contact dermatitis in Italy (1984-1993) by GIRDCA (Gruppo Italiano Ricerca Dermatiti da Contatto e Ambientali). Am J Contact Dermat. 10(1):18-30 (1999).

24. Osdoit S, Guillet MH, Guillet G. Contact sensitization to quaternium-15 acting as a warning sign for curare allergy. Contact Dermatitis. 65(2):120-2 (2011).

25. Park ME, Zippin JH. Allergic contact dermatitis to cosmetics. Dermatol Clin [Internet]. 32(1):1-11 (2014).

26. Suneja T, Belsito D V. Thimerosal in the detection of clinically relevant allergic contact reactions. J Am Acad Dermatol. 45(1):23-7 (2001).

27. Geier DA, King PG, Hooker BS, Dórea JG, Kern JK, Sykes LK, et al. Thimerosal: Clinical, epidemiologic and biochemical studies. Clin Chim Acta [Internet]. 444:212-20 (2015).

28. Yim E, Baquerizo Nole KL, Tosti A. Contact dermatitis caused by preservatives. Dermat contact, atopic, Occup drug [Internet]. 25(5):215-31 (2014).

29. Wolf $R$, Wolf $D$, Tüzün $B$, Tüzün $Y$. Contact dermatitis to cosmetics. Clin Dermatol. 19(4):50215 (2001).

30. Epstein JH. Phototoxicity and photoallergy. Vol. 18, Seminars in Cutaneous Medicine and Surgery. p. 274-84 (1999).

31. Lintner K, Genet V. A physical method for preservation of cosmetic products. Int J Cosmet Sci. 20(2):103-15 (1998).

32. Bandowe BAM, Bigalke M, Boamah L, Nyarko E, Saalia FK, Wilcke W. Polycyclic aromatic compounds (PAHs and oxygenated PAHs) and trace metals in fish species from Ghana (West Africa): Bioaccumulation and health risk assessment. Environ Int [Internet]. 65:135-46 (2014).

33. Zhang $Y$, Kim $C$, Zheng $T$. Hair dye use and risk of human cancer. Front Biosci (Elite Ed) [Internet]. 4:516-28 (2012). 